\title{
Survivin expression as an independent predictor of overall survival in malignant peritoneal mesothelioma
}

\author{
GUOZUN ZHANG $^{1 *}$, DONG-LIANG YANG ${ }^{2 *}$, GUOQI ZHENG ${ }^{1}$ and YUFEI LIANG ${ }^{1}$ \\ ${ }^{1}$ Department of Gastroenterology, Cangzhou Central Hospital; ${ }^{2}$ Department of Medical Statistics, \\ Cangzhou Medical College, Cangzhou, Hebei 061001, P.R. China
}

Received June 19, 2019; Accepted February 14, 2020

DOI: $10.3892 / 01.2020 .11505$

\begin{abstract}
Malignant peritoneal mesothelioma (MPeM) is an incurable cancer strongly associated with asbestos exposure and characterised by poor prognosis. The aim of the present study was to elucidate the prognostic and predictive value of CD146 and survivin expression in MPeM. Diagnostic biopsies from 60 patients with MPeM were collected and analysed for CD146, survivin and Ki-67 expression using immunohistochemistry. Complete clinical and follow-up information was obtained from patients' records. CD146 was expressed in 31/60 MPeM specimens and survivin in 34/60 specimens, with both expression levels being significantly associated with the Ki-67 labelling index (Ki-67LI). Kaplan-Meier and univariate Cox regression analyses revealed that a lower peritoneal cancer index (PCI), tumour-directed treatment, stage I, lower Ki-67LI and lower CD146 and survivin expression had a statistically positive effect on overall survival (OS). Cox regression analysis revealed that PCI [hazard ratio $(\mathrm{HR})=1.99 ; 95 \% \mathrm{CI}, 1.04-3.83$; $\mathrm{P}=0.038]$, survivin $(\mathrm{HR}=1.47 ; 95 \% \mathrm{CI}, 1.03-2.10 ; \mathrm{P}=0.034)$ and treatment protocol including intraperitoneal chemotherapy $(\mathrm{HR}=0.28 ; 95 \% \mathrm{CI}, 0.14-0.57 ; \mathrm{P}=0.013)$ and systemic chemotherapy $(\mathrm{HR}=0.13 ; 95 \% \mathrm{CI}, 0.04-0.42 ; \mathrm{P}=0.013)$ retained independent prognostic significance for OS. All of these were included in the nomogram. Calibration curves showed good agreement between nomogram-predicted and observed survival. The $\mathrm{C}$-index of the nomogram for predicting OS was 0.77. A lower PCI, intraperitoneal chemotherapy, systemic chemotherapy and a lower level of survivin were powerful prognostic markers in patients with MPeM. The proposed nomogram provides individual survival prediction for patients with MPeM.
\end{abstract}

Correspondence to: Professor Guoqi Zheng, Department of Gastroenterology, Cangzhou Central Hospital, 16 Xinhua West Road, Cangzhou, Hebei 061001, P.R. China

E-mail: zhengguoqi5502@126.com

*Contributed equally

Key words: peritoneal mesothelioma, survival, nomogram, immunohistochemistry, CD146, survivin

\section{Introduction}

Malignant mesothelioma ( $\mathrm{MM})$ is a rare but aggressive and fatal neoplasm that originates from the thoracic and abdominal serosal membranes, with malignant peritoneal mesothelioma (MPeM) representing 7-30\% of MM cases (1). Environmental and occupational exposure to asbestos is associated with this condition, and the incidence of MPeM in East China is 4.5 cases per million individuals in 2018 (2). Due to a lack of specificity of clinical symptoms, difficulty in making an early diagnosis and rapid disease progression with few effective treatments, the median survival prognosis is $\leq 12$ months from diagnosis (2). Recently, it has been shown that cytoreductive surgery (CRS), radiotherapy and chemotherapy could increase the survival of patients with MPeM (3). Therefore, early predictors of prognosis may help to guide intensive treatment protocols to improve survival and quality of life for patients with a short life expectancy. Due to the influence of tumour morphology and radiological markers, such as TNM stage, it is difficult to fully assess the prognosis of patients with MPeM.

$\mathrm{Ki}-67$ is a nuclear protein that is detected in the active phases of the cell cycle $\left(\mathrm{G}_{1}, \mathrm{G}_{2}, \mathrm{~S}\right.$ and $\left.\mathrm{M}\right)$ but absent in quiescent cells $\left(\mathrm{G}_{0}\right)$ (4). Therefore, Ki-67 is widely used as a predictive and prognostic marker in numerous types of cancer, such as those of the breast (5), lung (6) and prostate (7). Ki-67 has also been demonstrated to be a prognostic factor for MM (8).

CD146 is a multifunctional molecule that is involved in several physiological and pathological processes involving immunity and angiogenesis, and has also been found to serve a critical role in cancer progression (9). In a number of tumours, such as melanoma and gallbladder cancer $(10,11)$, CD146 has been found to promote cancer progression and migration. In malignant pleural mesothelioma, CD146 has been identified as an indicator of poor prognosis (12). However, only one study was found to report the effect of CD146 on the prognosis of MPeM (13).

Survivin is encoded by the baculoviral inhibitor of apoptosis repeat-containing 5 gene and is an important inhibitor of effector caspases in the apoptosis pathway. It is overexpressed in a number of tumours, but not in normal differentiated tissues, and may serve a key role in tumour prognosis $(14,15)$. Survivin is used to evaluate the prognosis of some tumours $(16,17)$, such as malignant pleural mesothelioma (18), but less so in MPeM. The present study aimed to explore the possible 
prognostic value of survivin and CD146 expression for patients with MPeM.

\section{Materials and methods}

Patients and tumour tissue samples. A total of 60 patients diagnosed with MPeM in Cangzhou Central Hospital (Cangzhou, China) over $\sim 3$ years (August 2015-September 2018) were included in the present study. The inclusion criteria were as follows: i) Newly diagnosed cases that were not combined with other tumours or other fatal diseases, such as severe infection, or failure of one or more organs that seriously affected survival; ii) patients with complete clinical data; and iii) patients without a history of receiving special medications, such as aspirin and diclofenac sodium. Patient demographics, asbestos exposure, treatments and follow-up data were retrieved from medical records. The histopathological diagnostic criteria of MPeM were established according to the guidelines for pathologic diagnosis of malignant mesothelioma (19). Yan et al (20) proposed a novel 'TNM' staging system in 2011, in which MPeM staging was determined according to the extent of peritoneal disease burden $(\mathrm{T})$, intra-abdominal nodal metastasis (N), extra-abdominal metastasis (M) and peritoneal carcinomatosis index (PCI). PCI was defined based on the following regions: The upper transverse plane, which is the lowest aspect of the costal margin; the lower transverse plane, which is the anterior superior iliac spine; and the abdomen, which is divided into three equal sectors by sagittal planes. The abdomen is divided into nine abdominopelvic regions (AR-8) by two transverse planes and two sagittal planes. AR-9 is located in the left upper abdomen, including the upper jejunum. AR-10 is the lower jejunum located in the left lower abdomen. AR-11 is the upper ileum located in the right upper abdomen, and AR-12 is the lower ileum, including the terminal ileum. For each region, four categories were used to estimate tumour volume: V0 indicated the absence of cancer at a particular abdominopelvic or anatomic site; V1 indicated tumour nodules $<0.5 \mathrm{~cm}$ in diameter (minimal volume); V2 indicated tumours $0.5-5 \mathrm{~cm}$ in diameter (moderate volume); and V3 indicated tumours $>5 \mathrm{~cm}$ in diameter (gross volume). Volume estimates were determined by the radiologist who performed the CT scan. PCI was based on lesion size (0-3) and tumour distribution (0-12) to determine the extent of the disease (0-39). PCI was calculated to determine the T stage, with scores of 1,2, 3 and 4 corresponding to PCI scores of 1-10, 11-20, 21-30 and 31-39, respectively. T1N0M0 was included in stage I disease; T2-3N0M0 represented stage II; and T4N0M0 and any N/M positive cases were classified as stage III.

Furthermore, 60 peritonitis tissues and 60 normal peritoneal tissues were selected as control specimen sets. The Pathology Department of Cangzhou Central Hospital provided tumour, peritonitis and peritoneal tissue specimens. Tumour and peritonitis tissue samples were obtained using ultrasound-guided biopsies for diagnostic purposes before patients received any clinical treatment. Normal mesothelial cells were taken from the normal peritoneal tissue of surgical peritoneal specimens.

Immunohistochemical analysis. Tissues were fixed in $4 \%$ phosphate-buffered paraformaldehyde at room temperature for $24 \mathrm{~h}$ and embedded in paraffin. Three consecutive
$4 \mu \mathrm{m}$-thick tissue sections of each paraffin block were used for immunohistochemical staining. Before dewaxing, the paraffin sections were rewarmed (baked in $70^{\circ} \mathrm{C}$ incubator for $2 \mathrm{~h})$. Paraffin sections were dewaxed and dehydrated, using xylene I and xylene II for $5 \mathrm{~min}$, and 100, 85 and 70\% ethanol for $3 \mathrm{~min}$, respectively. In a medical microwave oven, sodium citrate buffer solution $(0.01 \mathrm{~mol} / \mathrm{l}, \mathrm{pH} 6.0)$ was heated to $95^{\circ} \mathrm{C}$, in which specimens were incubated for $25 \mathrm{~min}$, and naturally cooled to room temperature (for $\sim 1 \mathrm{~h}$ ). Specimens were incubated in $0.03 \% \mathrm{H}_{2} \mathrm{O}_{2}$ at $37^{\circ} \mathrm{C}$ for 15 min to block endogenous peroxidase activity. Specimens were washed by $0.01 \mathrm{~mol} / 1$ PBS ( $\mathrm{pH}=7.4$ ) for $5 \mathrm{~min}$ between steps. Specimens were incubated with $50 \mathrm{ul}$ of normal 5-10\% goat serum blocking antigen for $15 \mathrm{~min}$ at room temperature. Specimens were incubated overnight at $4^{\circ} \mathrm{C}$ with primary antibodies against the following molecules: Survivin (dilution, 1:100; rabbit monoclonal, clone EP119; OriGene Technologies, Inc.; cat. no. S1130), CD146 (dilution, 1:100; rabbit monoclonal, clone EP54; OriGene Technologies, Inc.; cat. no. GTX34461) and Ki-67 (dilution, 1:200; rabbit monoclonal, clone EP5; OriGene Technologies, Inc.; cat.no. GTX16667). After incubation (at room temperature for $40 \mathrm{~min}$ ) with peroxidase-conjugated secondary antibodies (diluted concentration 1:200; Santa Cruz Biotechnology, Inc.), a Diaminobenzidine Peroxidase Substrate kit (Laboratories, Inc.) was used to visualise signals (light microscope; magnification, $x 43$; Olympus Corporation, put in multiple x10). Negative control specimens were processed under the same conditions, except that blocking liquid (negative control, the first antibody was replaced by normal serum and stained by immunohistochemistry S-P method) was used in place of the primary antibody.

Immunoreactivity evaluation. For evaluation, using the Jiangsu Jieda 801 image analysis system (Jetta Technology; version no. 801), >200 tumour cells were scored per field (magnification $x 40$ ). Sections of tumour tissues were scored semi-quantitatively for survivin and CD146 as follows: $0,<5 \%$ immuno-positive cells; $1+, 5-25 \%$ immuno-positive cells; $2+$, 26-50\% immuno-positive cells; $3+,>50 \%$ immuno-positive cells. Score 0 was defined as negative, and scores $1+, 2+$ and $3+$ were defined as positive, which corresponds to grade 4 expression. The Ki-67 labelling index (Ki-67LI) was determined by the number of positive cells per 500 tumour cells: Lower $\mathrm{Ki}-67 \mathrm{LI}, \leq 15 \%$; and higher $\mathrm{Ki}-67 \mathrm{LI},>15 \%$, which corresponds to grade 2 expression.

Statistical analysis. Correlations between parameters were tested by calculating the Spearman's rank correlation coefficient (rate). Kaplan-Meier analysis was used to calculate the overall cumulative probability of survival, and the log-rank test was used to assess differences in survival. Overall survival (OS) was measured from the date of initial diagnosis to the date of last follow-up examination or mortality (median). Univariate analysis was performed to assess the association between prognostic factors and survival (rate). Prognostic factors that were identified as significant in the univariate analysis were included in the multivariate analysis using the Cox proportional hazards model (rate). The nomogram was formulated using the 'rms' version 5.1-4 package in $\mathrm{R}$ version 3.5.2 software ( $\mathrm{R}$ Foundation for Statistical Computing; 
www.r-project.org) as a tool to predict the prognosis of MPeM and forest maps to show the hazard ratios (HRs) of independent prognostic factors. $\mathrm{P}<0.05$ was considered to indicate a statistically significant difference; however, $\mathrm{P}<0.10$ was considered statistically significant in univariate and multivariate analyses. The performance of the nomogram was estimated using a calibration curve. The predictive accuracy of the model was estimated using the concordance index (C-index). Statistical analyses were performed using SPSS v22.0 (IBM Corp.) and $\mathrm{R}$ version 3.5.2 software. Packages, including 'survival', 'nomogramEx', 'rms' and 'survminer' were used. The version number of survival, nomogramEx, rms and survminer respectively is $2.44-1,2.0,5.1-3$ and 0.4 .3 .

\section{Results}

Patients. In total, 60 patients were evaluated in the present study, comprising 22 men and 38 women (1:1.73). The median age at diagnosis was 62 years (range, 42-84). Asbestos exposure was documented for $86.7 \%$ of patients. Epithelioid and non-epithelioid tumours were found in 30 cases each (50\%). The mean PCI was 27.5 (range, 3-39). According to the novel 'TNM' staging system, five patients (8.3\%) had stage I, 47 patients (78.3\%) had stage II and eight patients (13.3\%) had stage III MPeM. A total of 38 patients received tumour-directed treatment with systemic or local abdominal chemotherapy, whereas the remaining patients received best supportive care (BSC), mainly due to comorbidities, advanced disease stage or poor performance status. The median OS was 9.25 months (range, 1-48 months). Five patients were still alive at the time of the final analysis. Clinical information is detailed in Table I.

Associations between survivin, CD146 and Ki-67 expression and clinicopathological parameters. Survivin and CD146 expression levels are detailed in Table I. Staining of CD146 was observed in the cytoplasm and cell membrane (Fig. 1A-H), and staining of survivin was observed in the nucleus and cytoplasm (Fig. 1I-P), while Ki-67 staining was only nuclear (Fig. 1Q-T). Carcinomas expressed survivin $(\geq 5 \%)$ in $34(56.67 \%)$ of 60 specimens, and CD146 $(\geq 5 \%)$ was detected in $31(51.67 \%)$ of 60 specimens. Spearman's rho analysis revealed that survivin and CD146 expression were both correlated with Ki-67LI ( $\mathrm{r}=0.425, \mathrm{P}=0.001$; $\mathrm{r}=0.362$, $\mathrm{P}=0.004$, respectively; Table II). Survivin, CD146 and $\mathrm{Ki}-67$ expression in normal mesothelium (Fig. 2A, C and E) and specimens from patients suffering from peritonitis (Fig. 2B, D and F) were negative. Mesothelial cells and lymphocytes, identified in peritonitis tissue specimens, exhibited no staining for the aforementioned proteins.

Survival analysis. Kaplan-Meier analysis and univariate Cox regression analysis demonstrated that a lower PCI, stage I, chemotherapy treatment (Fig. 3A-D; Table III) and a lower $\mathrm{Ki}-67 \mathrm{LI}$ had significantly positive effects on OS in patients with MPeM (Fig. 4A; Table III). In addition, a lower level of survivin expression was significantly associated with improved OS in grade 4 patients $(\mathrm{P}<0.000$; Fig. 4C; Table III), while high CD146 expression was associated with poor MPeM prognosis ( $\mathrm{P}=0.041$; Fig. 4B). All factors were included in the
Table I. Demographic patient characteristics $(n=60)$.

\begin{tabular}{|c|c|}
\hline Factors & Value or no. of patients \\
\hline \multicolumn{2}{|l|}{ Age, years } \\
\hline Median & 62 \\
\hline Range & $42-84$ \\
\hline \multicolumn{2}{|l|}{ Sex, $n$} \\
\hline Male & 22 \\
\hline Female & 38 \\
\hline \multicolumn{2}{|c|}{ Asbestos exposure, $\mathrm{n}$} \\
\hline+ & 52 \\
\hline- & 8 \\
\hline \multicolumn{2}{|c|}{ Histological type, $\mathrm{n}$} \\
\hline Epithelioid & 30 \\
\hline Non-epithelioid & 30 \\
\hline \multicolumn{2}{|l|}{ PCI, n } \\
\hline$\leq 30$ & 30 \\
\hline$>30$ & 30 \\
\hline \multicolumn{2}{|l|}{ TNM stage, $\mathrm{n}$} \\
\hline Stage I & 5 \\
\hline Stage II & 47 \\
\hline Stage III & 8 \\
\hline \multicolumn{2}{|l|}{ Treatment } \\
\hline BSC & 22 \\
\hline Chemotherapy & 38 \\
\hline \multicolumn{2}{|l|}{ Ki67 } \\
\hline$\leq 0.15$ & 30 \\
\hline$>0.15$ & 30 \\
\hline \multicolumn{2}{|l|}{ Survivin, $\mathrm{n}$} \\
\hline$<5 \%$ & 26 \\
\hline $5-25 \%$ & 21 \\
\hline $26-50 \%$ & 8 \\
\hline$>50 \%$ & 5 \\
\hline \multicolumn{2}{|l|}{ CD146, n } \\
\hline$<5 \%$ & 29 \\
\hline $5-25 \%$ & 18 \\
\hline $26-50 \%$ & 10 \\
\hline$>50 \%$ & 3 \\
\hline
\end{tabular}

PCI, peritoneal cancer index; BSC, best supportive care.

multivariate Cox analysis, in which a lower PCI $(\mathrm{HR}=1.99$; 95\% CI, 1.04-3.83; $\mathrm{P}=0.038)$, lower survivin expression $(\mathrm{HR}=1.47 ; 95 \% \mathrm{CI}, 1.03-2.10 ; \mathrm{P}=0.034)$, and treatment protocols, including intraperitoneal chemotherapy $(\mathrm{HR}=0.28$; 95\% CI, 0.14-0.57; $\mathrm{P}<0.001)$ and systemic chemotherapy $(\mathrm{HR}=0.13 ; 95 \% \mathrm{CI}, 0.04-0.42 ; \mathrm{P}<0.001)$ retained independent prognostic significance, with a positive effect on OS (Table III). TNM stage $(\mathrm{P}=0.123)$ was also included in the forest maps (with $\mathrm{P}=0.05$ as the cut-off point), and the results are shown in Fig. 4D. High PCI and expression of survivin indicated poor prognosis in patients with MPeM. Intraperitoneal and systemic chemotherapy had a statistically positive effect on overall 


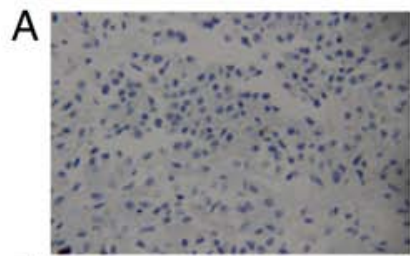

E

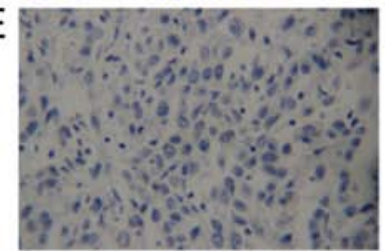

I
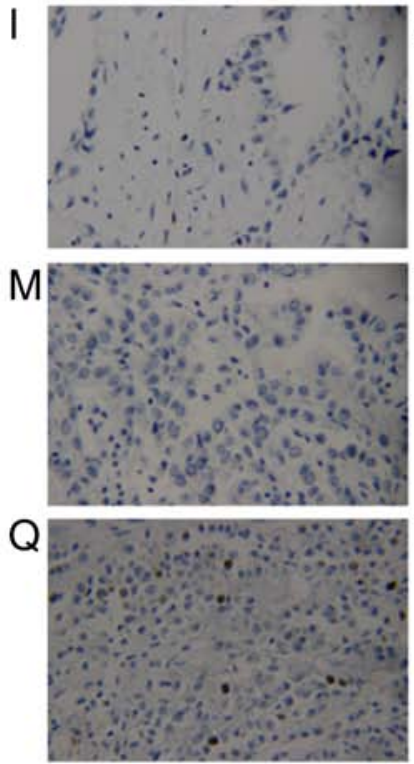

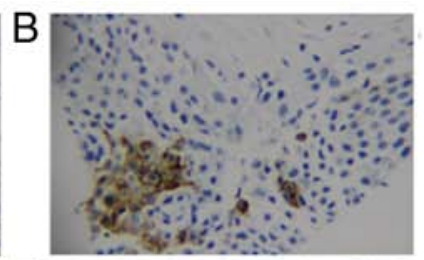

$\mathrm{F}$
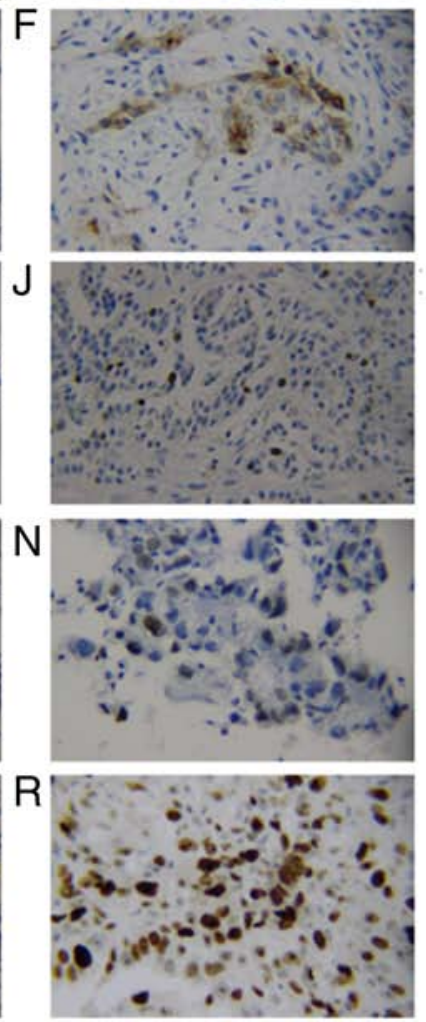
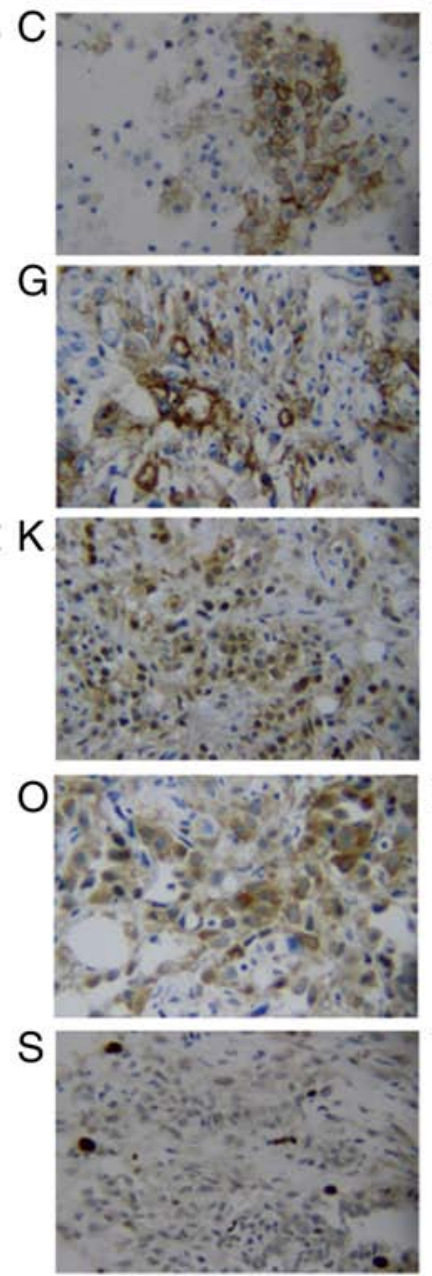

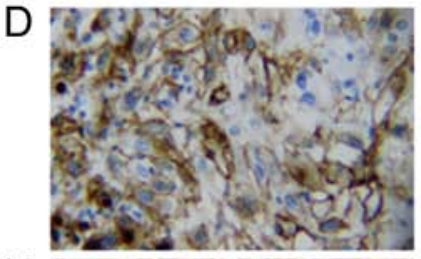

$\mathrm{H}$
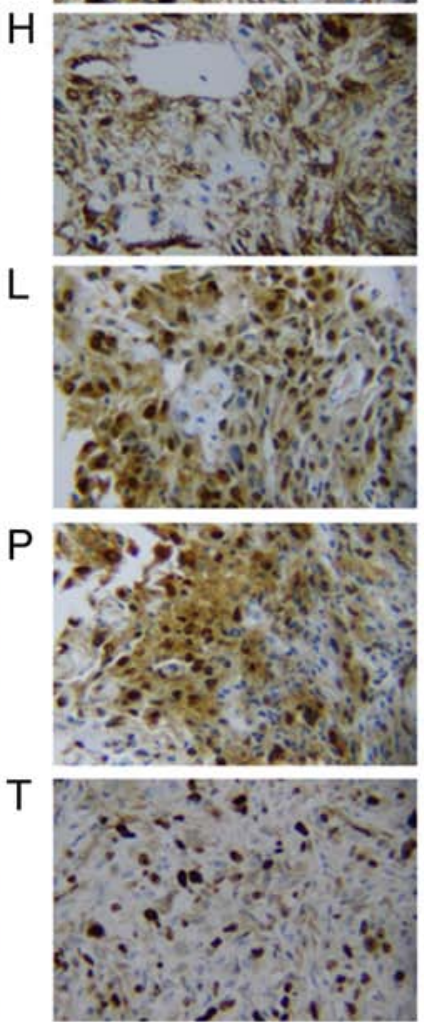

Figure 1. Representative IHC images of CD146, survivin and Ki-67 expression. Expression of CD146 in epithelioid MPeM: (A) Negative; (B) 1+; (C) 2+; (D) 3+. Expression of CD146 in non-epithelioid MPeM: (E) Negative; (F) 1+; (G) 2+; (H) 3+. Expression of survivin in epithelioid MPeM: (I) Negative; (J) 1+; (K) 2+; (L) 3+. Expression of survivin in non-epithelioid MPeM: (M) Negative; (N) 1+; (O) 2+; (P) 3+. Expression of Ki-67 in epithelioid MPeM: (Q) Lower expression; (R) higher expression. Expression of Ki-67 in non-epithelioid MPeM: (S) Lower expression; (T) higher expression. Staining of CD146 was observed in the cytoplasm and cell membrane, staining of survivin was found in the nucleus and cytoplasm, while Ki-67 staining was nuclear. Magnification, $\mathrm{x} 400$. IHC, immunohistochemistry; MPeM, malignant peritoneal mesothelioma.

survival. Treatment protocol was a disordered multivariable, and treatment protocols $=0$ was the reference.

Construction and validation of the nomogram. The graphical calculator or nomogram uses line scores to assist the clinician in quickly estimating individualised patient-specific OS (Fig. 5A). PCI, TNM stage, survivin and treatment protocols were incorporated into the calculator. PCI was divided into two categories: $\leq 30$ and $>30$; TNM stage was divided into three categories: Stage I, stage II and stage III; survivin was divided into four categories: $<5 \%, 5-25 \%, 26-50 \%$ and $>50 \%$; treatment protocols were divided into three categories: BSC, intraperitoneal chemotherapy and systemic chemotherapy. The model determined the estimated values of 1-, 2- and 3-year OS by simply adding up the corresponding scores of the four factors to calculate the total score. In addition, the performance of the nomogram was graphically evaluated using a calibration curve (Fig. 5B-D). The C-index was 0.77, and the predicted line overlapped well with the reference line, demonstrating good performance of the nomogram.

\section{Discussion}

The worldwide incidence of MPeM continues to rise, partly because of its association with asbestos exposure. Although MPeM is traditionally considered resistant to antitumour therapy, some patients exhibit a good response to CRS with hyper-thermic intraperitoneal chemotherapy (HIPEC) or multidisciplinary therapy (3). Therefore, it is important to identify prognostic factors that can predict who will benefit from these treatments. Some of the predictive factors for OS in patients with MPeM include age, sex, histologic type and grade, lymphatic metastasis, and imaging staging $(2,15,21)$. Clinical imaging examinations mainly include CT and MRI, which do not convey pathological information and cannot fully assess the prognosis of MPeM. Individual studies have also identified blood neutrophil-to-lymphocyte ratio (22) and glucose transporter 1 expression (23) as predictors of survival.

Numerous studies (24-26) have suggested that age is a prognostic factor for MPeM, and older age suggests poor prognosis. In general, the prognosis of patients over 65 is worse than that of patients under 65 years of age (27). Research has 
Table II. Association and differences of survivin and CD146 expression with clinicopathologic parameters and Ki-67LI in patients with malignant peritoneal mesothelioma.

\begin{tabular}{|c|c|c|c|c|c|c|c|c|c|c|c|}
\hline \multirow[b]{3}{*}{ Variable } & \multirow[b]{3}{*}{ No. } & \multicolumn{5}{|c|}{ Survivin } & \multicolumn{5}{|c|}{ CD146 } \\
\hline & & \multicolumn{4}{|c|}{ Reactive grade, $\mathrm{n}$} & \multirow[b]{2}{*}{ P-value } & \multicolumn{4}{|c|}{ Reactive grade, $\mathrm{n}$} & \multirow[b]{2}{*}{ P-value } \\
\hline & & 0 & $1+$ & $2+$ & $3+$ & & 0 & $1+$ & $2+$ & $3+$ & \\
\hline \multicolumn{12}{|l|}{ Age (years) } \\
\hline$\leq 62$ & 31 & 14 & 10 & 3 & 4 & 1.000 & 14 & 11 & 5 & 1 & 0.881 \\
\hline$>62$ & 29 & 12 & 11 & 5 & 1 & & 15 & 7 & 5 & 2 & \\
\hline \multicolumn{12}{|l|}{ Sex } \\
\hline Male & 22 & 11 & 4 & 5 & 2 & 0.400 & 10 & 7 & 4 & 1 & 0.774 \\
\hline Female & 38 & 15 & 17 & 3 & 3 & & 19 & 11 & 6 & 2 & \\
\hline \multicolumn{12}{|l|}{ Histologicaltype } \\
\hline Epithelioid & 30 & 11 & 12 & 5 & 2 & 0.424 & 12 & 11 & 6 & 1 & 0.322 \\
\hline Non-epithelioid & 30 & 15 & 9 & 3 & 3 & & 17 & 7 & 4 & 2 & \\
\hline \multicolumn{12}{|l|}{ Ki67 } \\
\hline$\leq 0.15$ & 30 & 19 & 8 & 2 & 1 & 0.001 & 20 & 6 & 4 & 0 & 0.004 \\
\hline$>0.15$ & 30 & 7 & 13 & 6 & 4 & & 9 & 12 & 6 & 3 & \\
\hline
\end{tabular}
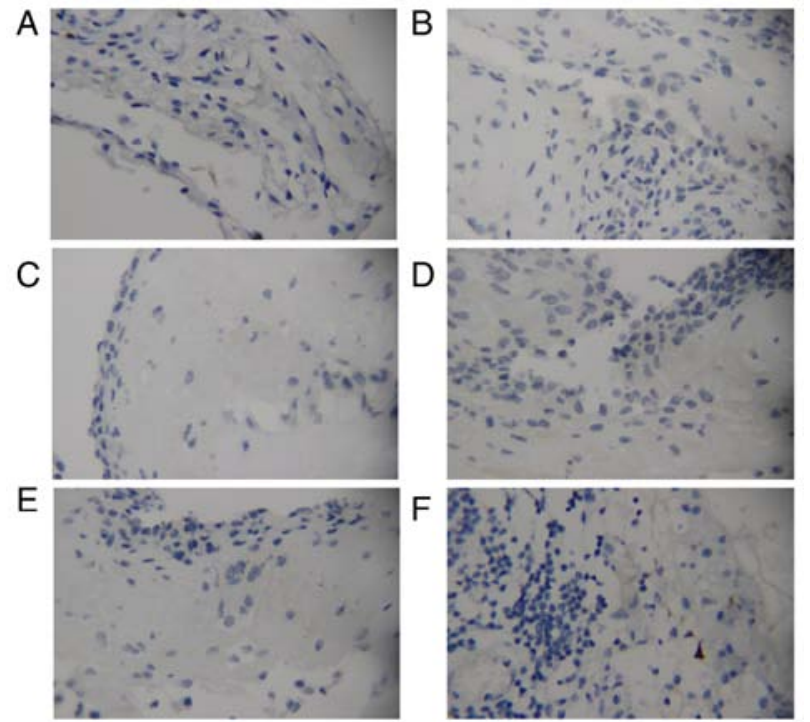

Figure 2. Survivin (A) CD146 (C) and Ki-67 (E) expression is absent in normal mesothelium. Survivin (B) CD146 (D) and Ki-67 (F) expression is absent in peritonitis specimens (B, D, F). Magnification x400.

revealed that sex and clinical stage are independent prognostic factors for OS in malignant pleural mesothelioma (28). MPeM can be divided into epithelioid, sarcomatoid and biphasic types; among the aforementioned, the epithelioid subtype is associated with an improved outcome. Prognosis of the non-epithelioid subtypes (including sarcomatoid and biphasic) is extremely poor $(24,26)$. The results of the present study demonstrated that age, sex and histopathological typing were not associated with MPeM prognosis using a univariate analysis. In future studies, the sample size could be increased and further research should be conducted to confirm the association between age, histological type and prognosis. Among the patients in the present study, $63.3 \%$ of the patients were female, the asbestos exposure rate was $86.7 \%$ in all patients included. In the present study, the high asbestos exposure rate and the frequent incidence of disease in women could be attributed to the practice of hand-spinning asbestos yarn in the 1970s, when the workers were mainly teenage girls.

Yan et al (20) created a clinicopathologic staging system that emphasizes the prognostic importance of tumour volume and distribution within the peritoneal cavity, lymph node involvement and extra-abdominal metastases. A high PCI has been shown to be a poor prognostic factor in MPeM in a study (20). In the present study, Kaplan-Meier and univariate Cox regression analyses showed that both a lower PCI and stage I tumours had significantly positive effects on OS, while multivariate Cox regression analysis did not confirm the associations between stage I and OS. All patients in the present study received internal conservative treatment, and the PCI and TNM grades were based on CT imaging, which may be different from previous surgical grades. In future studies, the sample size and treatment methods should be improved to verify the aforementioned results.

Research has suggested that CRS combined with HIPEC should be considered as standard treatment for patients diagnosed with MPeM (29). For numerous patients with MPeM who are unable to tolerate or unwilling to undergo surgery, clinicians usually provide supportive treatment or chemotherapy. Currently, the first-line clinical systemic therapy is pemetrexed combined with cisplatin or carboplatin (30). In the present study, tumour-directed treatment, especially systemic chemotherapy with pemetrexed alone or in combination with cisplatin and intraperitoneal chemotherapy with cisplatin, had a significantly positive effect on OS in MPeM.

$\mathrm{Ki}-67$ is an indicator of tumour replication. High expression of Ki-67 indicates active tumour growth. Numerous studies $(27,31)$ have indicated that high levels of Ki-67 result 
Table III. Univariate and multivariate analyses of factors affecting OS in patients with malignant peritoneal mesothelioma.

\begin{tabular}{|c|c|c|c|c|c|c|}
\hline \multirow[b]{2}{*}{ Variable } & \multicolumn{3}{|c|}{ Univariate analysis } & \multicolumn{3}{|c|}{ Multivariate analysis } \\
\hline & Hazard ratio & $95 \% \mathrm{CI}$ & P-value & Hazard ratio & $95 \% \mathrm{CI}$ & P-value \\
\hline Sex (female vs. male) & 0.66 & $(0.37-1.17)$ & 0.110 & & & \\
\hline Age ( $\leq 62$ vs. $>62)$ & 1.00 & $(0.59-1.70)$ & 0.990 & & & \\
\hline Asbestos exposure (yes vs. no) & 0.96 & $(0.43-2.14)$ & 0.900 & & & \\
\hline $\begin{array}{l}\text { Histological type } \\
\text { (Epithelioid vs. Non-epithelioid) }\end{array}$ & 1.21 & $(0.70-2.09)$ & 0.450 & & & \\
\hline PCI ( $\leq 30$ vs. $>30)$ & 2.18 & $(1.26-3.76)$ & 0.001 & 1.99 & $(1.04-3.83)$ & 0.044 \\
\hline TNM Stage (I vs. II vs. III) & 1.61 & $(1.05-2.73)$ & 0.047 & 1.56 & $(0.89-2.76)$ & 0.123 \\
\hline Treatment (BSC vs. chemotherapy treatment) & 0.30 & $(0.16-0.57)$ & $<0.000$ & & & \\
\hline Ki-67 ( $\leq 15 \%$ vs. $>15 \%)$ & 2.19 & $(1.24-3.89)$ & 0.007 & & & \\
\hline Survivin (negative vs. positive) & 1.65 & $(1.17-2.32)$ & $<0.000$ & 1.47 & $(1.03-2.10)$ & 0.034 \\
\hline CD146 (negative vs. positive) & 1.48 & $(1.08-2.03)$ & 0.041 & & & \\
\hline Treatment protocol & & & $<0.000$ & & & $<0.001$ \\
\hline Best supportive care & 1.00 & & & 1.00 & & \\
\hline Intraperitoneal chemotherapy & 0.32 & $(0.17-0.62)$ & & 0.28 & $(0.14-0.57)$ & $<0.001$ \\
\hline Systemic chemotherapy & 0.20 & $(0.07-0.59)$ & & 0.13 & $(0.04-0.42)$ & $<0.001$ \\
\hline
\end{tabular}

PCI, peritoneal cancer index; BSC, best supportive care.

A
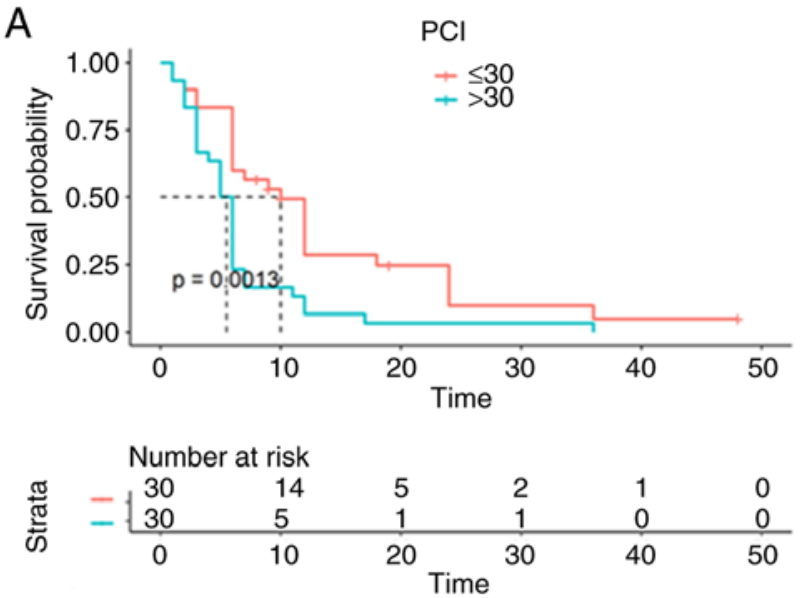

C
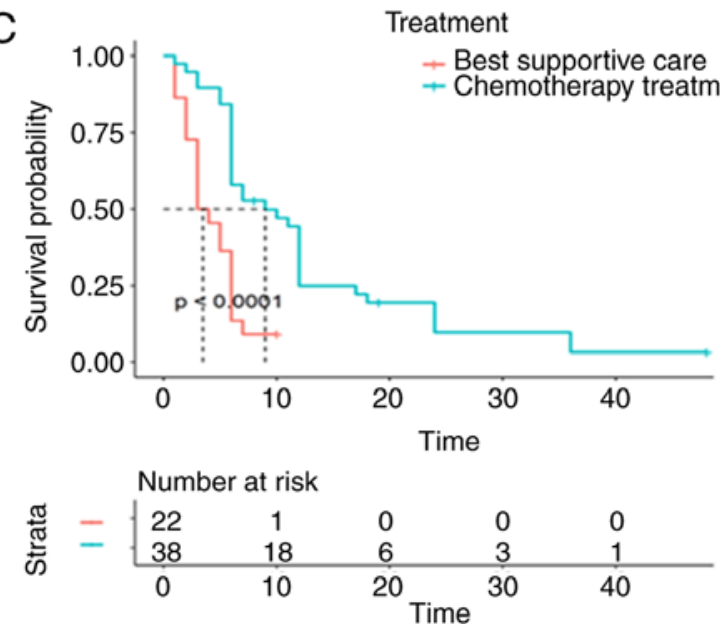

B
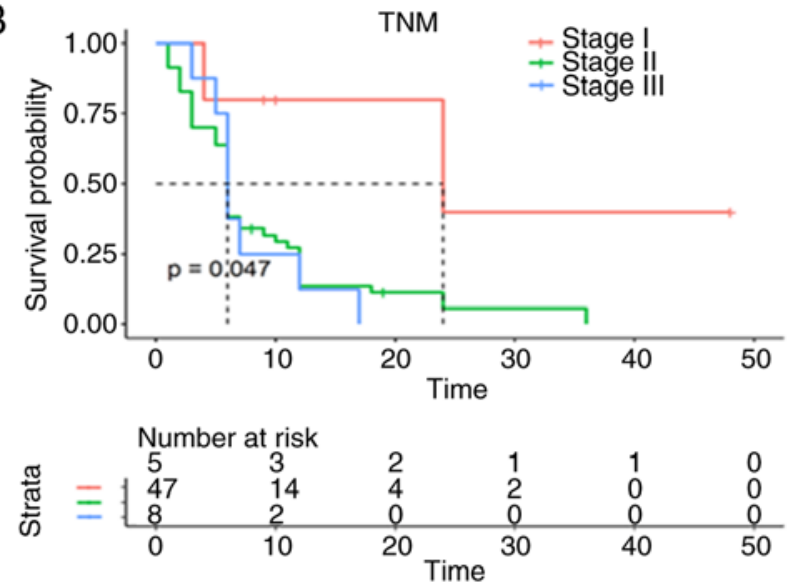

D

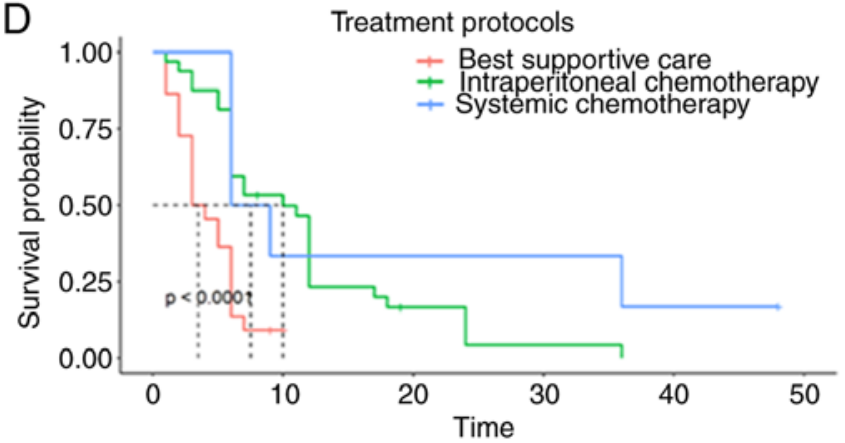

Number at risk

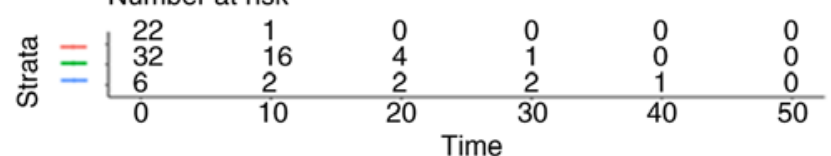

Figure 3. Kaplan-Meier curves of the overall survival rates in patients with MPeM according to different predictors. (A) PCI; (B) T stage; (C) grade 2 treatment protocols; (D) grade 3 treatment protocols. All predictors were statistically significant (P-values are shown in the figure). Time-dependent numbers at risk are listed at the bottom. Dashed lines represent median survival. PCI, peritoneal cancer index; MPeM, malignant peritoneal mesothelioma. 

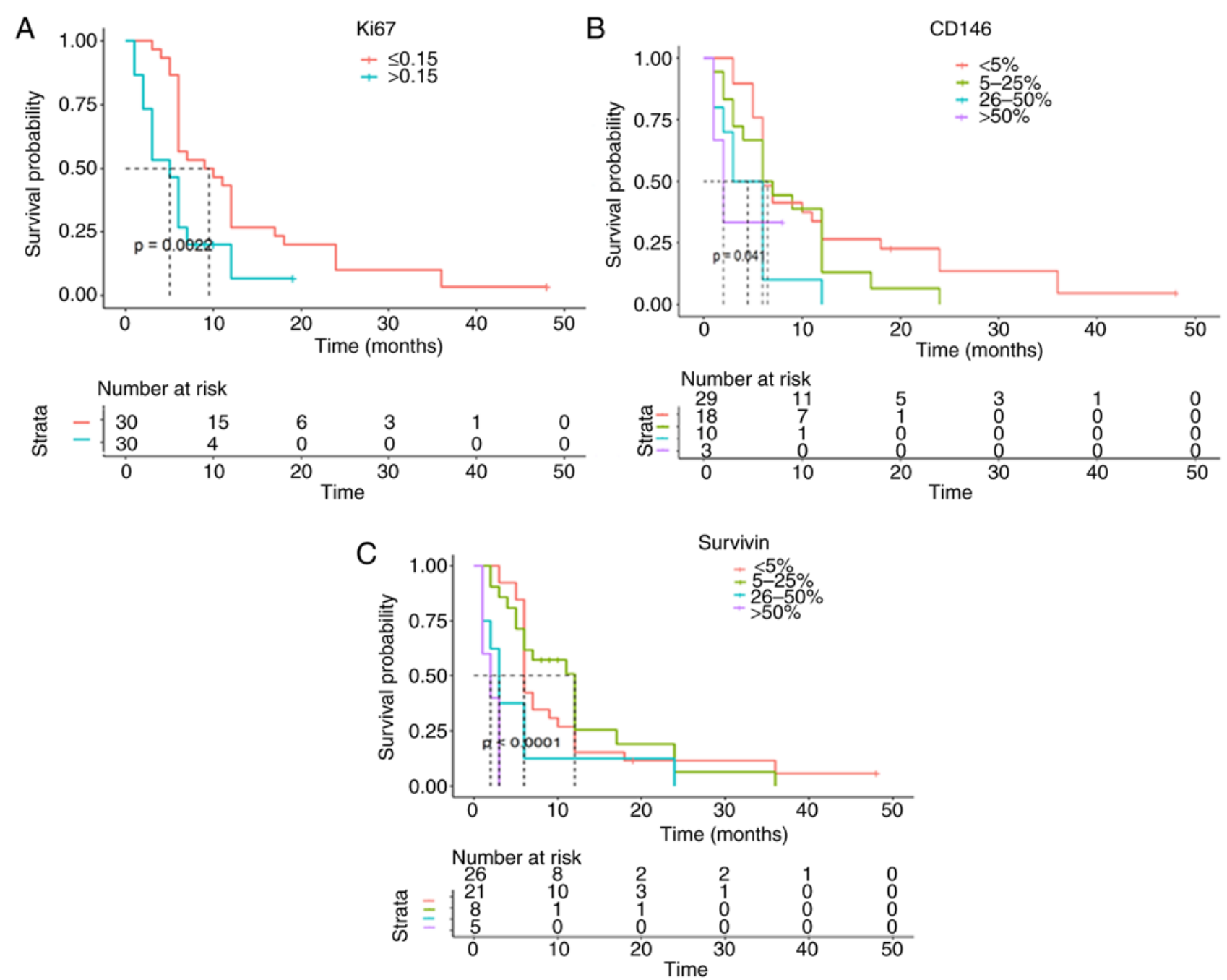

D

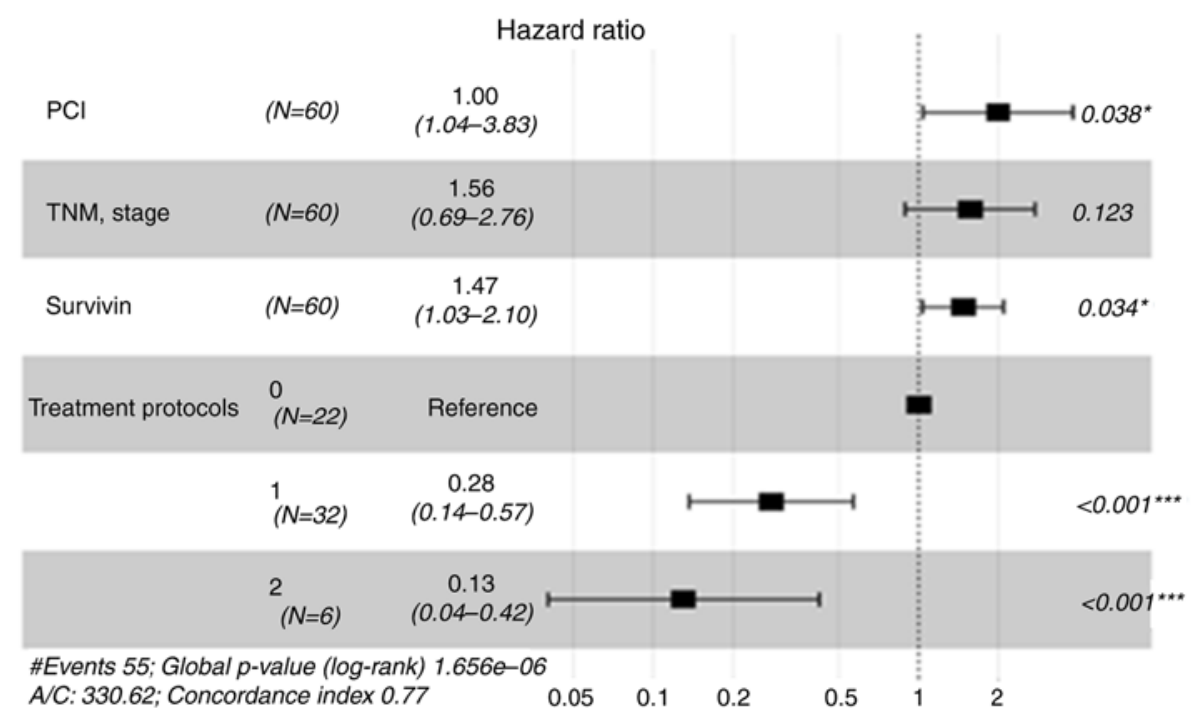

Figure 4. Kaplan-Meier survival curves for different predictors. (A) Ki-67 grade 2 expression; (B) CD146 grade 4 expression; (C) survivin grade 4 expression; (D) forest plot of disease-free survival hazard ratios of major subgroups (exploratory analysis). Dashed lines represent median survival. PCI, peritoneal cancer index. Treatment protocols 0 , best supportive care; Treatment protocols 1 , intraperitoneal chemotherapy; Treatment protocols 2 , systemic chemotherapy. ${ }^{*} \mathrm{P}<0.05$. ${ }^{* * *} \mathrm{P}<0.001$.

in a poor prognosis. Patients with MPeM with high Ki-67 expression and a high PCI have an average survival time of 10 months (20). A multicentre study reported that the Ki-67 index is an independent prognostic factor for epithelioid rather than non-epithelioid malignant pleural mesothelioma (8). Pillai et al (32) examined the expression of Ki-67 in $42 \mathrm{MPeM}$ tumours and concluded that high $\mathrm{Ki}-67$ expression is associated with poor survival. In the present study, Ki-67 expression 
A

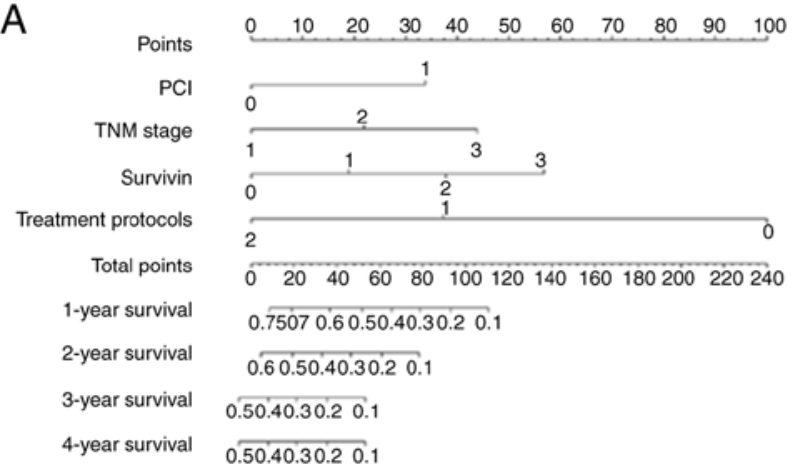

C

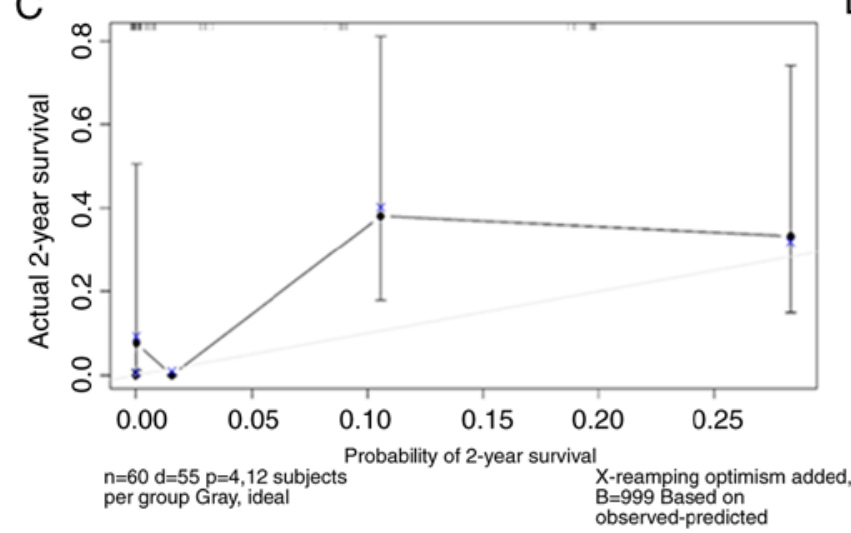

B
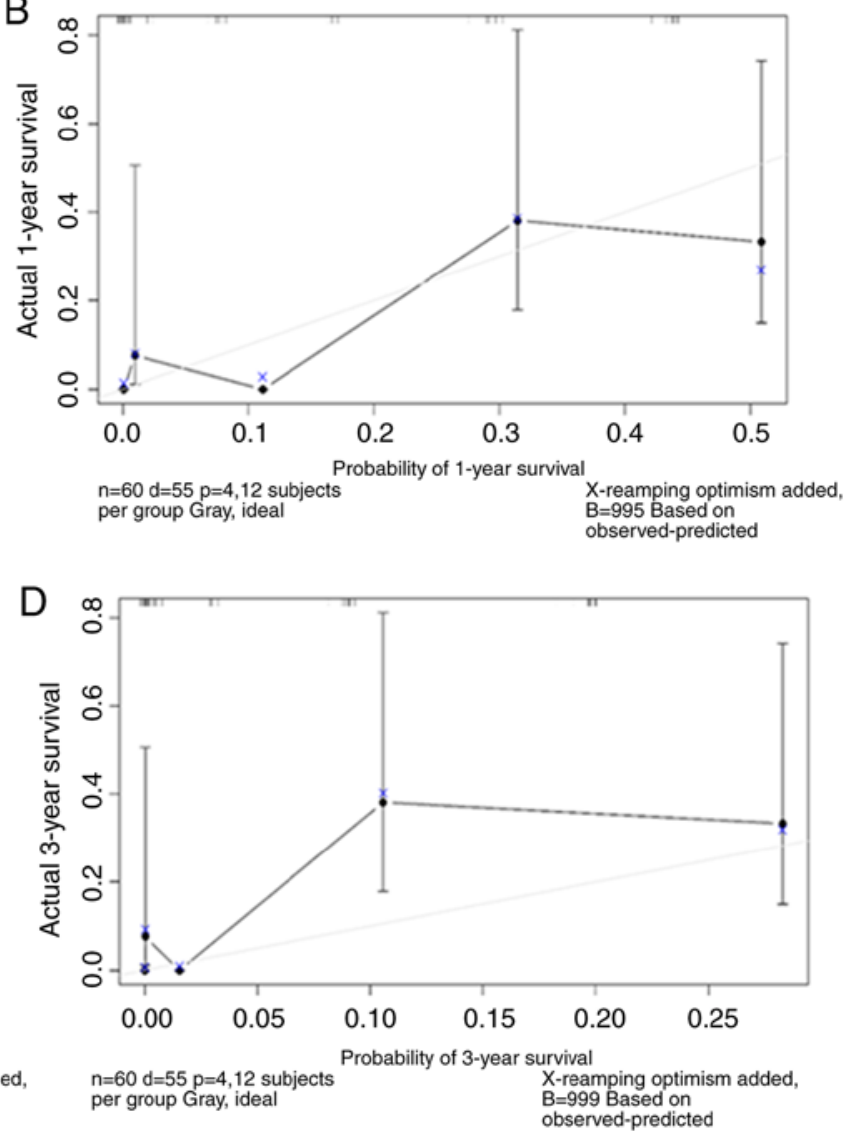

Figure 5. Nomogram figure and calibration curves used to predict patient survival rates. (A) Nomogram to predict the survival time of patients with MPeM. Patients' PCI are located in the row labelled 'PCI', and a straight line is drawn up to the row labelled 'Points' to determine the corresponding points. This process is then repeated for each of the remaining factors. After the total points are summed, a straight line is drawn from the appropriate total point number location to the rows labelled '1 year survival', '2 year survival' and ' 3 year survival' to predict patient survival probability. Calibration curves for predicting (B) 1-, (C) 2- and (D) 3 year OS for patients with MPeM. Actual survival measured via Kaplan-Meier analysis is shown on the Y-axis, and the nomogram-predicted survival is shown on the X-axis. Calibration curves showed good performance between nomogram-predicted and observed survival. The C-index of the nomogram for predicting OS was 0.77 . OS, overall survival; PCI, peritoneal cancer index; MPeM, malignant peritoneal mesothelioma.

was tumour-specific and was negative in normal mesothelium and peritonitis specimens. Univariate analysis showed that lower Ki-67 expression suggested an improved prognosis in patients with MPeM, while multivariate analysis did not confirm this association.

CD146 is a cell adhesion molecule that participates in several physiological and pathological processes, such as signal transduction, cell migration, angiogenesis and immune responses. It has become an increasingly important molecule, especially as a novel biomarker for angiogenesis and cancer. Zeng et al (33) found that CD146 expression is significantly associated with late stage tumours and poor prognosis in breast cancer. In addition, CD146 is significantly associated with advanced tumour stage in malignant melanoma (34) and mesothelioma (35). The results of the present study revealed that CD146 expression was correlated with $\mathrm{Ki}-67$ expression and that there was no CD146 expression in mesothelial cells and peritonitis tissues. Moreover, univariate analysis suggested that lower CD146 expression was associated with improved prognosis in patients with MPeM. These results suggested that CD146 was related to the prognosis of MPeM, but this correlation was not confirmed using the multivariate analysis.
A number of studies have shown that the expression of nuclear survivin is related to cell proliferation, advanced disease and poor clinical outcome $(18,27)$. Overexpression of survivin suggests poor prognosis in numerous types of cancer, such as gallbladder cancer and pancreatic ductal adenocarcinoma $(36,37)$. In addition, elevated concentrations of survivin in pleural fluid are associated with shorter survival in patients with malignant pleural effusion (16). Meerang et al (38) reported that high survivin labelling index is an indicator of poor prognosis in patients with malignant pleural mesothelioma. However, other studies identified no association between survivin and disease outcome $(39,40)$.

At present, there have only been a few reports on the association between survivin and MPeM prognosis $(41,42)$. The present study revealed that survivin was expressed in $34 / 60(56.7 \%)$ mesothelioma specimens in a tumour-specific manner. Spearman's rho analysis revealed a significant correlation between survivin expression and $\mathrm{Ki}-67 \mathrm{LI}(\mathrm{r}=0.425$; $\mathrm{P}=0.001$ ), confirming that survivin expression was associated with cell proliferation, which was consistent with the results of Bitanihirwe et al (43). Furthermore, the univariate and multivariate analyses showed that a lower level of survivin expression was significantly associated with improved OS 
$(\mathrm{P}<0.0001 ; \mathrm{P}=0.034)$. The aforementioned results suggest that survivin is important in predicting the prognosis of MPeM. Other studies have shown that patients with MM with an improved response to tumour-directed treatment have higher survivin expression in their tumours $(20,44)$. This indicator may offer theoretical guidance for providing improved clinical treatment for different patients.

The present study constructed a clinical assessment tool (nomogram) with a high accuracy $(\mathrm{C}$-index, 0.77$)$ for predicting survival in patients with MPeM. This tool provided a simple graphical display of predicted survival based on clinical parameters that affect MPeM OS: PCI, TNM stage, survivin and treatment protocols. Estimation of survival may individualise patient treatment and follow-up, including influencing the extent of surgical and systemic therapy, and the frequency of diagnostic imaging. This model should be validated with additional data and in a prospective manner, and future refinement will likely improve its predictive accuracy.

In conclusion, PCI, tumour-directed treatment (including intraperitoneal and systemic chemotherapy), and survivin levels appear to serve vital roles in influencing survival time. These factors were incorporated into a nomogram to predict outcomes in MPeM, providing a useful tool for clinicians to personalise treatment in this poor prognosis population.

\section{Acknowledgements}

The authors would like to thank Professor Mark Abramovitz for editing the English language of a draft of this manuscript.

\section{Funding}

The present study was funded by Cang Zhou Finance Bureau (grant no. 1213018ZD).

\section{Availability of data and materials}

The datasets used and/or analysed during the current study are available from the corresponding author on reasonable request.

\section{Authors' contributions}

GZha and GZhe conceived and designed the study. DLY and YFL analysed and interpreted the data. GZha wrote, edited and reviewed the manuscript. All authors gave final approval for publication. GZha takes full responsibility for the work as a whole, including the study design, access to data and the decision to submit and publish the manuscript. All authors read and approved the final manuscript.

\section{Ethics approval and consent to participate}

Thepresentstudy wasapprovedby theMedicalEthicsCommittee of CangZhou Central Hospital (approval no. 2012-012-01) and was carried out according to the Declaration of Helsinki. All participants signed informed consent.

\section{Patient consent for publication}

Not applicable.

\section{Competing interests}

The authors declare that they have no competing interests.

\section{References}

1. Van Zandwijk N, Clarke C, Henderson D, Musk AW, Fong K, Nowak A, Loneragan R, McCaughan B, Boyer M, Feigen M, et al: Guidelines for the diagnosis and treatment of malignant pleural mesothelioma. J Thorac Dis 5: E254-E307, 2013.

2. Hui S, Guo-Qi Z, Xiao-Zhong G, Chun-Rong L, Yu-Fei L and Dong-Liang-Y: IMP3 as a prognostic biomarker in patients with malignant peritoneal mesothelioma. Hum Pathol 81: 138-147, 2018 .

3. Alexander HR, Bartlett DL, Pingpank JF, Libutti SK, Royal R, Hughes MS, Holtzman M, Hanna N, Turner K, Beresneva T and Zhu Y: Treatment factors associated with long-term survival after cytoreductive surgery and regional chemotherapy for patients with malignant peritoneal mesothelioma. Surgery 153: 779-786. 2013

4. Gerdes J, Li L, Schlueter C, Duchrow M, Wohlenberg C, Gerlach C, Stahmer I, Kloth S, Brandt E and Flad HD: Immunobiochemical and molecular biologic characterization of the cell proliferation-associated nuclear antigen that is defined by monoclonal antibody Ki-67. Am J Pathol 138: 867-873, 1991.

5. Cuzick J, Dowsett M, Pineda S, Wale C, Salter J, Quinn E, Zabaglo L, Mallon E, Green AR, Ellis IO, et al: Prognostic value of a combined estrogen receptor, progesterone receptor, $\mathrm{Ki}-67$, and human epidermal growth factor receptor $2 \mathrm{immu}-$ nohistochemical score and comparison with the genomic health recurrence score in early breast cancer. J Clin Oncol 29: 4273-4278, 2011

6. Yang J, Ramnath N, Moysich KB, Asch HL, Swede H, Alrawi SJ, Huberman J, Geradts J, Brooks JS and Tan D: Prognostic significance of MCM2, Ki-67 and gelsolin in non-small cell lung cancer. BMC Cancer 6: 203, 2006.

7. Zellweger T, Günther S, Zlobec I, Savic S, Sauter G, Moch H, Mattarelli G, Eichenberger T, Curschellas E, Rüfenacht $\mathrm{H}$, et al: Tumour growth fraction measured by immunohistochemical staining of Ki67 is an independent prognostic factor in preoperative prostate biopsies with small-volume or low-grade prostate cancer. Int J Cancer 124: 2116-2123, 2009.

8. Ghanim B, Klikovits T, Hoda MA, Lang G, Szirtes I, Setinek U, Rozsas A, Renyi-Vamos F, Laszlo V, Grusch M, et al: Ki67 index is an independent prognostic factor in epithelioid but not in non-epithelioid malignant pleural mesothelioma: A multicenter study. Br J Cancer 112: 783-792, 2015.

9. Jiang G, Zhang L, Zhu Q, Bai D, Zhang C and Wang X: CD146 promotes metastasis and predicts poor prognosis of hepatocellularcarcinoma. J Exp Clin Cancer Res 35: 38, 2016.

10. Lei X, Guan CW, Song Y and Wang H: The multifaceted role of CD146/MCAM in the promotion of melanoma progression. Cancer Cell Int 15: 3, 2015.

11. Wang W, Yang ZL, Liu JQ, Jiang S and Miao XY: Identification of CD146 expression, angiogenesis, and lymphangiogenesis as progression, metastasis and poor-prognosis related markers for gallbladder adenocarcinoma. Tumour Biol 33: 173-182, 2012.

12. Sato A, Torii I, Okamura Y, Yamamoto T, Nishigami T, Kataoka TR, Song M, Hasegawa S, Nakano T, Kamei T and Tsujimura T: Immunocytochemistry of CD146 is useful to discriminate between malignant pleural mesothelioma and reactive mesothelium. Mod Pathol 23: 1458-1466, 2010.

13. Okazaki Y, Nagai H, Chew SH, Li J, Funahashi S, Tsujimura T and Toyokuni S: CD146 and insulin-like growth factor 2 mRNA-binding protein 3 predict prognosis of asbestos-induced rat mesothelioma. Cancer Sci 104: 989-995, 2013.

14. Goričar K, Kovač V, Franko A, Dodič-Fikfak M and Dolžan V: Serum surviving levels and outcome of chemotherapy in patients with maligna-nt mesothelioma. Dis Markers 2015: 316739, 2015.

15. Mita AC, Mita MM, Nawrocki ST and Giles FJ: Survivin: Key regulator of mitosis and apoptosis and novel target for cancer therapeutics. Clin Cancer Res 15: 5000-5005, 2008.

16. Arellano-Orden E, Romero-Romero B, Sánchez-López V, Martín-Juan J, Rodríguez-Panadero F and Otero-Candelera R: Survivin is a negative prognostic factor in malignant pleural effusion. Eur J Clin Invest 48: 10, 2018. 
17. Contis J,Lykoudis PM, Goula K, Karandrea D and Kondi-Pafiti A: Survivinn expression as an independent predictor of overall survival in pancreatic adenocarcinoma. J Cancer Res Ther 14 (Suppl): S719-S723, 2018

18. Gordon GJ, Mani M, Mukhopadhyay L, Dong L, Edenfield HR, Glickman JN, Yeap BY, Sugarbaker DJ and Bueno R: Expression patterns of inhibitor of apoptosis proteins in malignant pleural mesothelioma. J Pathol 211: 447-454, 2007.

19. Husain AN, Colby T, Ordonez N, Krausz T, Attanoos R, Beasley MB, Borczuk AC, Butnor K, Cagle PT, Chirieac LR, et al: Guidelines for pathologic diagnosis of malignant mesothelioma: 2012 update of the consensus statement from the international mesothelioma interest group. Arch Pathol Lab Med 137: 647-667, 2013.

20. Yan TD, Deraco M, Elias D, Glehen O, Levine EA, Moran BJ Morris DL, Chua TC, Piso P, Sugarbaker PH, et al: A novel tumor-node-metastasis (TNM) staging system of diffuse malignant peritoneal mesothelioma using outcome analysis of a multi-institutional database. Cancer 117: 1855-1863, 2011.

21. Vigneswaran WT, Kircheva DY, Ananthanarayanan V, Watson S, Arif Q, Celauro AD, Kindler HL and Husain AN: Amount of epithelioid differentiation is a predictor of survival in malignant pleural mesothelioma. Ann Thorac Surg 103: 962-966, 2017.

22. Yin W, Zheng G, Yang K, Song H and Liang Y: Analysis of prognostic factors of patients with malignant peritoneal mesothelioma. World J Surg Oncol 16: 44, 2018.

23. Hommell-Fontaine J, Isaac S, Passot G, Decullier E, Traverse-Glehen A, Cotte E, You B, Mohamed F, Gilly FN, Glehen $\mathrm{O}$ and Berger F: Malignant peritoneal mesothelioma treated by cytoreductive surgery and hyperthermic intraperitoneal chemotherapy: Is GLUT1 expression a major prognostic factor? A preliminary study. Ann Surg Oncol 20: 3892-3898, 2013.

24. Verma V, Sleightholm RL, Rusthoven CG, Koshy M, Sher DJ, Grover S and Simone CB II: Malignant peritoneal mesothelioma: National practice patterns, outcomes, and predictors of survival. Ann Surg Oncol 25: 2018-2026, 2018.

25. Shavelle R, Vavra-Musser K, Lee J and Brooks J: Life expectancy in pleural and peritoneal mesothelioma. Lung Cancer Int 2017: 2782590, 2017.

26. Leinwand JC, Taub RN, Chabot JA and Kluger MD: Two-Stage cytoreductive surgery and intraperitoneal chemotherapy for diffuse malignant peritoneal mesothelioma: Predictors of overal survival in an intention-to-treat series. Ann Surg Oncol Dec 12: $10,2019$.

27. Magge D, Zenati MS, Austin F, Mavanur A, Sathaiah M, Ramalingam L, Jones $\mathrm{H}$, Zureikat AH, Holtzman $M$, Ahrendt S, et al: Malignant peritoneal mesothelioma: Prognostic factors and oncologic outcome analysis. Ann Surg Oncol 21: $1159-1165,2014$

28. Takamori S, Toyokawa G, Shimokawa M, Kinoshita F, Kozuma Y, Matsubara T, Haratake N, Akamine T, Hirai F, Seto T, et al: The c-reactive protein/albumin ratio is a novel significant prognostic factor in patients with malignant pleural mesothelioma: A retrospective multi-institutional study. Ann Surg Oncol 25: $1555-1563,2018$

29. Helm JH, Miura JT, Glenn JA, Marcus RK, Larrieux G, Jayakrishnan TT, Donahue AE, Gamblin TC, Turaga KK and Johnston FM: Cytoreductive surgery and hyperthermic intraperitoneal chemotherapy for malignant peritoneal mesothelioma: A systematic review and meta-analysis. Ann Surg Oncol 22: $1686-1693,2015$

30. Levý M, Boublíková L, Büchler T and Šimša J: Treatment of malignant peritoneal mesothelioma. Klin Onkol 32: 333-337, 2019.
31. Feldman AL, Libutti SK, Pingpank JF, Bartlett DL, Beresnev TH Mavroukakis SM, Steinberg SM, Liewehr DJ, Kleiner DE and Alexander HR: Analysis of factors associated with outcome in patients with malignant peritoneal mesothelioma undergoing surgical debulking and intraperitoneal chemotherapy. J Clin Oncol 21: 4560-4567, 2003.

32. Pillai K, Pourgholami MH, Chua TC and Morris DL: Ki67-BCL2 index in prognosis of malignant peritoneal mesothelioma. Am J Cancer Res 3: 411-423, 2013.

33. Zeng Q, Li W, Lu D, Wu Z, Duan H, Luo Y, Feng J, Yang D, Fu L and Yan X: CD146, an epithelial-mesenchymal transition inducer, is associated with triple-negative breast cancer. Proc Natl Acad Sci USA 109: 1127-1132, 2012.

34. Liu Q, Zhang B, Zhao X, Zhang Y, Liu Y and Yan X: Blockade of adhesion molecule CD146 causes pregnancy failure in mice. J Cell Physiol 215: 621-626, 2008.

35. Bidlingmaier S, He J, Wang Y, An F, Feng J, Barbone D, Gao D, Franc B, Broaddus VC and Liu B: Identification of MCAM/CD146 as the target antigen of a human monoclonal antibody that recognizes both epithelioid and sarcomatoid types of mesothelioma. Cancer Res 69: 1570-1577, 2009.

36. Nigam J, Chandra A, Kazmi HR, Singh A, Gupta V, Parmar D and Srivastava MK: Expression of serum survivin protein in diagnosis and prognosis of gallbladder cancer: A comparative study. Med Oncol 31: 167, 2014.

37. Ren YQ, Zhang HY, Su T, Wang XH and Zhang L: Clinical significance of serum survivin in patients with pancreatic ductal adenocarcinoma. Eur Rev Med Pharmacol Sci 18: 3063-3068, 2014.

38. Meerang M, Bérard K, Friess M, Bitanihirwe BK, Soltermann A, Vrugt B, Felley-Bosco E, Bueno R, Richards WG, Seifert B, et al: Low merlin expression and high survivin labeling index are indicators for poor prognosis in patients with malignant pleural mesothelioma. Mol Oncol 10: 1255-1265, 2016.

39. Hmeljak J, Erčulj N, Dolžan V, Kern I and Cör A: BIRC5 promoter SNPs do not affect nuclear survivin expression and survival of malignant pleural mesothelioma patients. J Cancer Res Clin Oncol 137: 1641-1651, 2011.

40. Kleinberg L, Lie AK, Flørenes VA, Nesland JM and Davidson B: Expression of inhibitor-of-apoptosis protein family members in malignant mesothelioma. Hum Pathol 38: 986-994, 2007.

41. De Cesare M, Cominetti D, Doldi V, Lopergolo A, Deraco M, Gandellini P, Friedlander S, Landesma Y, Kauffman MG, Shacham S, et al: Anti-Tumor activity of selective inhibitors of XPO1/CRM1-mediated nuclear export in diffuse malignant peritoneal mesothelioma: The role of survivin. Oncotarget 6 : 13119-13132, 2015

42. Zaffaroni N, Costa A,Pennati M, De Marco C, Affini E, Madeo M, Erdas R, Cabras A, Kusamura S and Baratti D: Survivin is highly expressed and promotes cell survival in malignant peritoneal mesothelioma. Cell Oncol 29: 453-466, 2007.

43. Bitanihirwe BK, Meerang M, Friess M, Soltermann A, Frischknecht L, Thies S, Felley-Bosco E, Tsao MS, Allo G, de Perrot M, et al: PI3K/mTOR signaling in mesothelioma patients treated with induction chemotherapy followed by extrapleural pneumonectomy. J Thorac Oncol 9: 239-247, 2014.

44. Hmeljak J, Erčulj N, Dolžan V, Pižem J, Kern I, Kovač V, Cemažar $M$ and Cör A: Is survivin expression prognostic or predictive in malignant pleural mesothelioma. Virchows Arch 462: 315-321, 2013.

This work is licensed under a Creative Commons Attribution-NonCommercial-NoDerivatives 4.0 International (CC BY-NC-ND 4.0) License. 